Symmetric-Key Encryption Based on Bioaffinity Interactions

Leif K. McGoldrick, Elizabeth A. Weiss, Jan Halámek*

Department of Chemistry, University at Albany, State University of New York, 1400

Washington Ave, Albany, New York, 12222, United States 
Supporting Information

Experimental Details: Encryption and Decryption Assays

It is important to note that although this paper was performed using the following parameters, those are not the only parameters that may be chosen for this process of encryption. Any reasonable setup of possible concentrations, temperatures, and other parameters may be used in order to heighten security and avoid making the same key twice. The only possible other change would be in the values at table 1, considering that those values are based off of standard deviation. This is due to higher deviations at other possible parameters due to the nature of enzyme assays. As these three assays are commonly used and well-studied, no unexpected or unusually high safety hazards were encountered.

For all of these assays, a Spectramax 384 Plus spectrophotometer was used. This instrument's monochromator allows for $1 \mathrm{~nm}$ increments to be used with $\pm 1 \mathrm{~nm}$ accuracy and 0.2 $\mathrm{nm}$ repeatability for the wavelength and a resolution of 0.001 OD with \pm 0.006 OD accuracy for microplate. A 96-well microtiter plate was used for all experimental runs as well. All runs were performed at $37^{\circ} \mathrm{C}$, measuring every 30 seconds. A carbonate buffer system was used for all assays, made from ultrapure water $(>18.2 \mathrm{M} \Omega \cdot \mathrm{cm})$ from an ELGA PURELAB flex water purification system.

As seen in Figure 2 of the main text, there are three assays being performed: alkaline phosphatase (ALP), lysozyme, and horseradish peroxidase (HRP). These assays each require certain substrates in order to work.

For the ALP assay, $50 \mathrm{mU}$ of ALP were used, alongside differing concentration of the only substrate, pNPP, at concentrations of $0.1,0.5$, and $5 \mathrm{mM}$. In this assay, the shake function of the instrument was used before the first scan and between consecutive scans ( 5 seconds before, 3 seconds between). This assay was measured at $405 \mathrm{~nm}$.

For the lysozyme assay, $2 \mathrm{U}$ of the enzyme were used along with varying concentration of Micrococcus lysodeikticus cells in a $0.1,0.2$, and $0.25 \mathrm{mg} / \mathrm{mL}$ cell suspensions. This enzyme involves the breaking down of these cells, so a decrease in absorbance was observed at $450 \mathrm{~nm}$.

For the HRP assay the each well consisted of $2.5 \mathrm{mU}$ HRP and different sets of concentrations for TMB and $\mathrm{H}_{2} \mathrm{O}_{2}: 0.25 \mathrm{mM} / 0.025 \mathrm{mM}, 0.5 \mathrm{mM} / 0.05 \mathrm{mM}$, and $1.5 \mathrm{mM} / 0.15$ $\mathrm{mM}$, respectively to build the corresponding assay graph in Figure 4c. This assay was measured at $650 \mathrm{~nm}$.

The average standard deviation of all assays is \pm 0.01913 OD with a maximum value for standard deviation of \pm 0.06 OD for ALP and HRP, and \pm 0.03 OD for Lysozyme. 
In order to better show the deviations of the graphs used in figure 4a-c, the graphs resulting from each of the three assays are included here, with smaller data points to aid with clarity. The average standard deviation for each graph is as follows: ALP: 0.02 OD, Lysozyme: 0.003 OD and HRP: 0.04 .
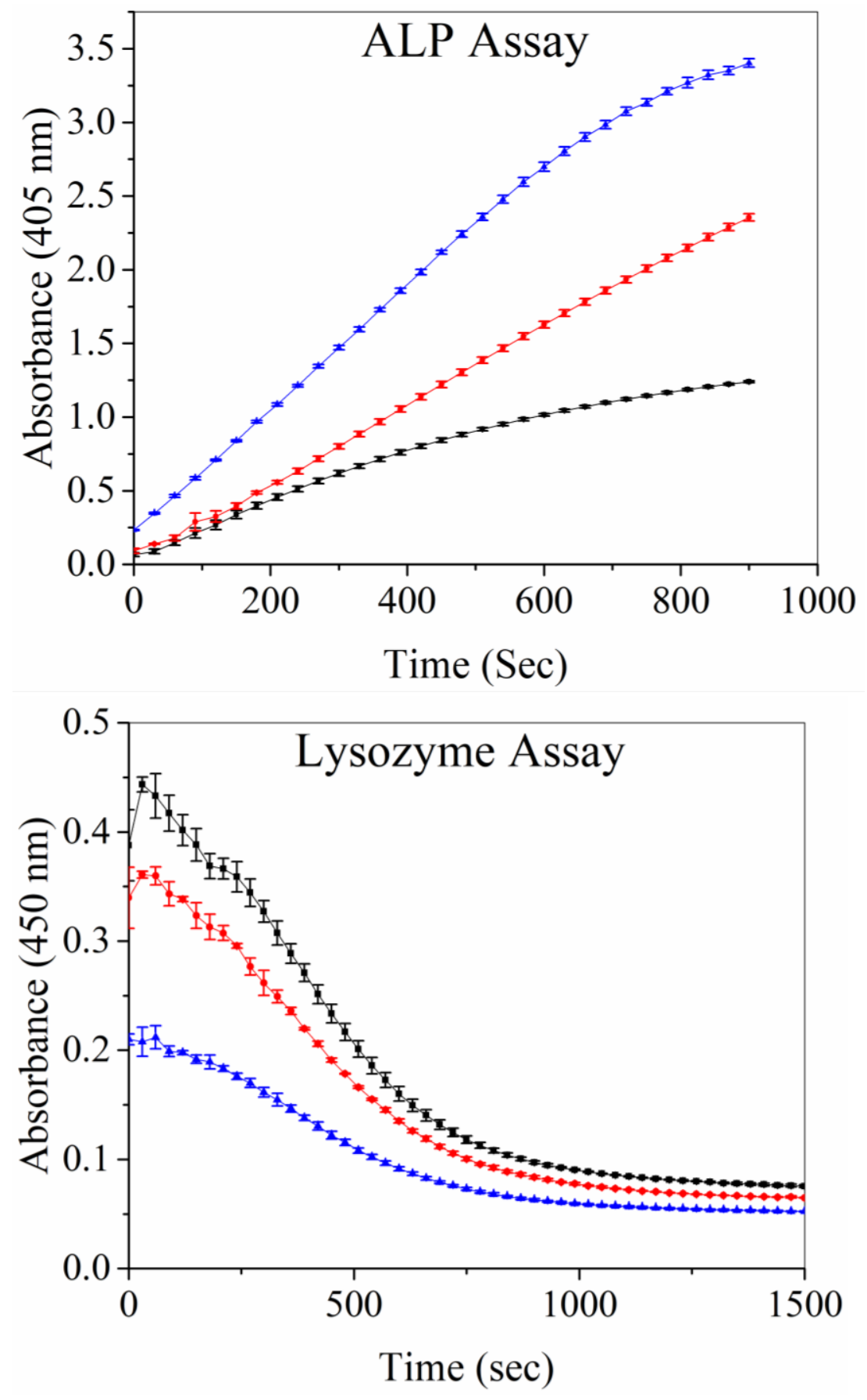


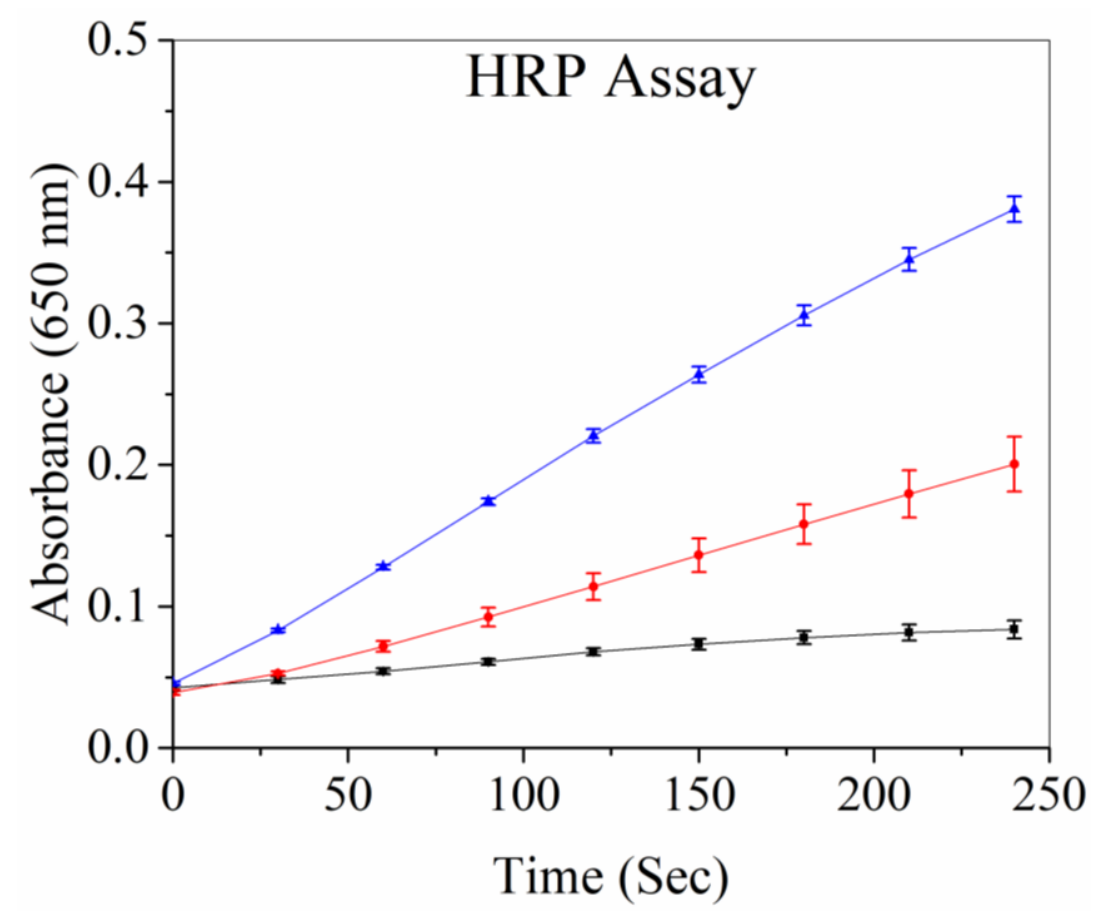

For further illustration of the effects of two other possible rotors, a change in $\mathrm{pH}$ for a single buffer (citrate) and different temperatures were measured using the HRP assay seen in figure 3 of the manuscript. $\mathrm{n}=3$
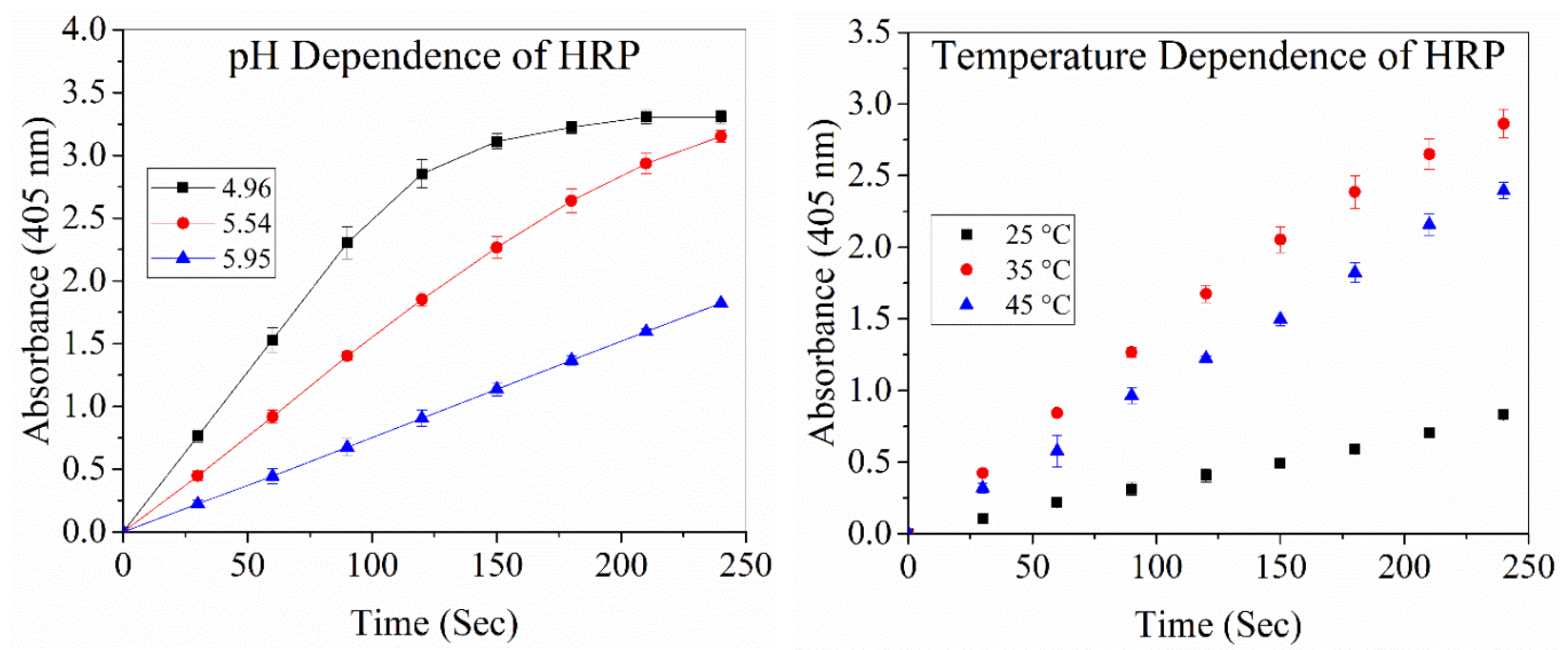
In order to show another enzyme that may be used for this methodology, the CK/PK/LDH (citrate kinase, pyruvate kinase, lactate dehydrogenase) enzyme cascade is shown below. This shows more complex assays, namely cascades, can also be used as they do provide reproducible results and vary. The following shows the changing of CK only for many concentrations of enzyme (concentrations in U/L). An accompanying figure detailing the assay is also below. $n=3$
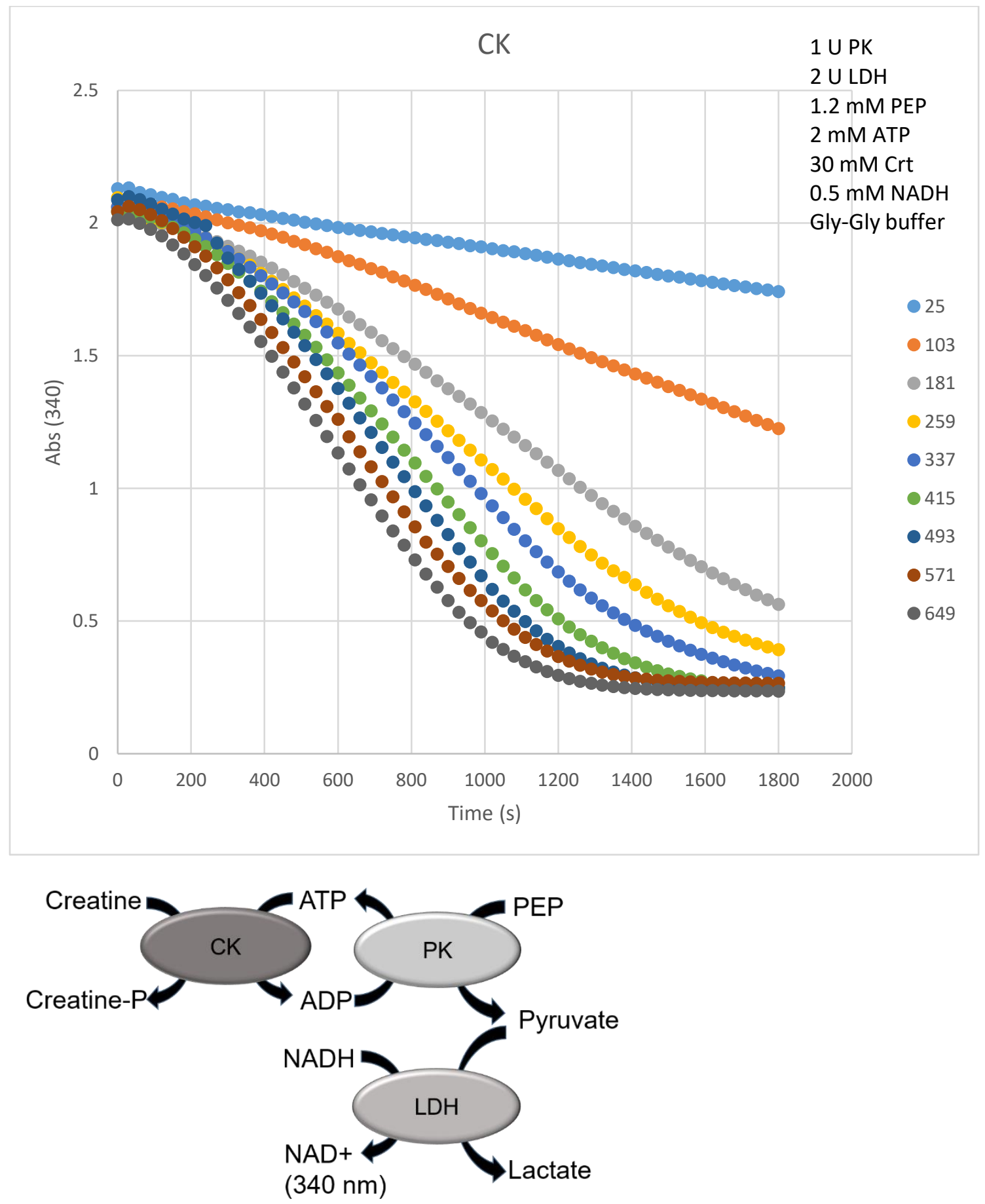


\section{Additional Messages.}

Three additional messages can be seen below to demonstrate different keys brought about by changing the experimental parameters using this methodology. For each of these messages, there will be a set of graphs for the assay(s) used for encryption and decryption as well as three tables. The three tables consist of: the encryption process, direct translation of the ciphertext according to table $1 \mathrm{~A}$ in the manuscript, and the decryption process, respectively. Each of these experiments were performed at different parameters including, but not limited to: using different amounts of enzyme and substrate, differing temperature, and differing sampling rate for the instrument. The assays used include the three from the paper: HRP, ALP, and Lysozyme (Lys), in addition to the $\mathrm{CK} / \mathrm{PK} / \mathrm{LDH}$ enzymatic cascade seen above. The corresponding data points in the graphs for the following messages are marked as stars and are red for encryption and green for decryption. The parameters for each of the assays are listed, but are not the only available sets for this methodology. N.B. sampling rate is shows as data point per amount of seconds (1 point/x sec)

\section{Extra message 1: SEND HELP}

HRP assay only

$2.5 \mathrm{mU}$ HRP, $2 \mathrm{mM}$ substrates. $40^{\circ} \mathrm{C}$, point/20 sec, $10 \mathrm{~min}$, Citrate Buffer $\mathrm{pH}$ 5.54, $405 \mathrm{~nm}$

\begin{tabular}{|l|l|l|l|l|l|}
\hline Letter & $\begin{array}{l}\text { Numerical } \\
\text { (Table 1A) }\end{array}$ & $\begin{array}{l}\text { Time } \\
\text { point } \\
(\mathrm{Sec})\end{array}$ & $\begin{array}{l}\text { Enc. } \\
\text { Key } \\
(+)\end{array}$ & Ciphertext (raw) & $\begin{array}{l}\text { Sent } \\
\text { ciphertext }\end{array}$ \\
\hline $\mathrm{S}$ & 2.99 & 20 & 0.2062 & 3.1962 & 3.20 \\
\hline $\mathrm{E}$ & 0.65 & 80 & 0.3243 & 0.9743 & 0.97 \\
\hline $\mathrm{N}$ & 6.38 & 120 & 0.4040 & 6.7840 & 6.78 \\
\hline $\mathrm{D}$ & 3.25 & 180 & 0.5248 & 3.7748 & 3.77 \\
\hline Space & 7.15 & 220 & 0.6096 & 7.7546 & 7.76 \\
\hline $\mathrm{H}$ & 4.03 & 260 & 0.6894 & 4.9194 & 4.72 \\
\hline $\mathrm{E}$ & 0.65 & 360 & 0.8907 & 1.5407 & 1.54 \\
\hline $\mathrm{L}$ & 4.81 & 460 & 1.0964 & 5.9064 & 5.91 \\
\hline $\mathrm{P}$ & 2.47 & 520 & 1.2083 & 3.6783 & 3.68 \\
\hline
\end{tabular}

\begin{tabular}{|l|l|}
\hline Ciphertext & $\begin{array}{l}\text { Direct } \\
\text { translation }\end{array}$ \\
\hline 3.20 & D \\
\hline 0.97 & R \\
\hline 6.78 & - \\
\hline 3.77 & G \\
\hline 7.76 & 2 \\
\hline 4.72 & L \\
\hline 1.54 & Y \\
\hline 5.91 & V \\
\hline 3.68 & G \\
\hline
\end{tabular}

\begin{tabular}{|l|l|l|l|}
\hline Ciphertext & $\begin{array}{l}\text { Dec. } \\
\text { Key } \\
(-)\end{array}$ & Plaintext & $\begin{array}{l}\text { Decrypted } \\
\text { message }\end{array}$ \\
\hline 3.20 & 0.2188 & 2.9812 & S \\
\hline 0.97 & 0.3297 & 0.6403 & E \\
\hline 6.78 & 0.4103 & 6.3697 & N \\
\hline 3.77 & 0.5348 & 3.2352 & D \\
\hline 7.76 & 0.6185 & 7.1415 & Space \\
\hline 4.72 & 0.7027 & 4.0179 & H \\
\hline 1.54 & 0.9060 & 0.6340 & E \\
\hline 5.91 & 1.1063 & 4.8437 & L \\
\hline 3.68 & 1.2253 & 2.4547 & P \\
\hline
\end{tabular}
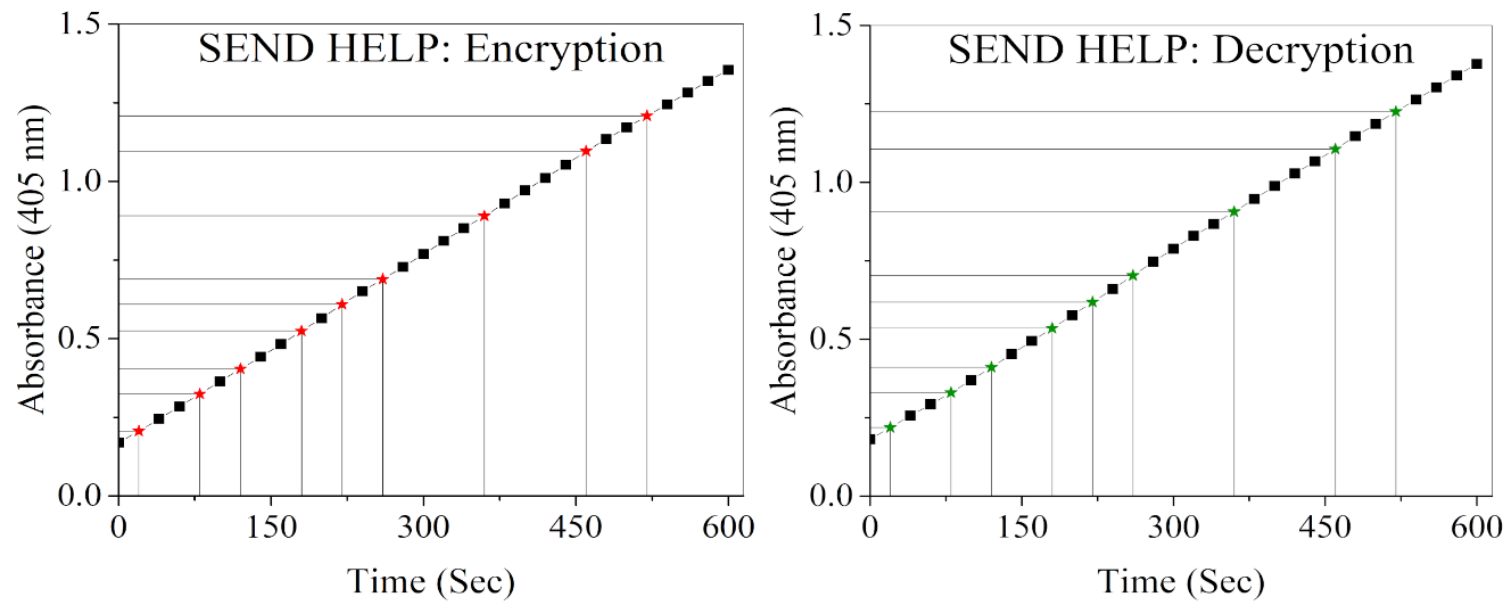
Extra message 2: SUNY ALBANY

CK/PK/LDH, HRP, and Lys

CK: 337 U CK, 1 U PK, 2 U LDH, 1.2 mM PEP, 2 mM ATP, 30 mM Crt, 0.5 mM NADH, GlyGly buffer $\mathrm{pH}$ 7.98, $30 \mathrm{~min}, 340 \mathrm{~nm}$

HRP: 5 mU, 3 mM substrates, Citrate Buffer pH 5.54, 10 min, 405 nm

Lys: $300 \mathrm{U}, 0.2 \mathrm{mg} / \mathrm{mL}$ cells, Citrate Buffer $\mathrm{pH} 5.54$, cut at plateau at $2.5 \mathrm{~min}, 450 \mathrm{~nm}$

All: $37^{\circ} \mathrm{C}$, point $/ 30 \mathrm{sec}$

\begin{tabular}{|l|l|l|l|r|r|}
\hline Letter & $\begin{array}{l}\text { Numerical } \\
\text { (Table 1A) }\end{array}$ & $\begin{array}{l}\text { Time } \\
\text { point } \\
\text { (Sec) }\end{array}$ & $\begin{array}{l}\text { Enc. } \\
\text { Key } \\
(+)\end{array}$ & Ciphertext (raw) & $\begin{array}{l}\text { Sent } \\
\text { ciphertext }\end{array}$ \\
\hline S & 2.99 & CK 420 & 1.7557 & 4.7457 & 4.75 \\
\hline U & 1.69 & CK 600 & 1.5218 & 3.2118 & 3.21 \\
\hline N & 6.38 & CK 780 & 1.2417 & 7.6217 & 7.62 \\
\hline Y & 1.43 & CK 1020 & 0.8703 & 2.3003 & 2.30 \\
\hline Space & 7.15 & HRP 90 & 0.8997 & 8.0497 & 8.05 \\
\hline A & 2.73 & HRP 270 & 2.1224 & 4.8524 & 4.85 \\
\hline L & 4.81 & HRP 450 & 3.0492 & 7.8592 & 7.86 \\
\hline B & 6.11 & HRP 540 & 3.2828 & 9.3928 & 9.39 \\
\hline A & 2.73 & Lys 30 & 0.2791 & 3.0091 & 3.01 \\
\hline N & 6.38 & Lys 60 & 0.2271 & 6.6071 & 6.61 \\
\hline Y & 1.43 & Lys 120 & 0.1974 & 1.6274 & 1.63 \\
\hline & & & & & \\
\hline
\end{tabular}

\begin{tabular}{|c|l|}
\hline Ciphertext & $\begin{array}{l}\text { Direct } \\
\text { translation }\end{array}$ \\
\hline $4.75 \mathrm{~L}$ \\
\hline $3.21 \mathrm{D}$ \\
\hline 7.62 & 2 \\
\hline $2.30 \mathrm{O}$ \\
\hline 8.05 & 3 \\
\hline $4.85 \mathrm{~L}$ \\
\hline 7.86 & 3 \\
\hline 9.39 & 9 \\
\hline $3.01 \mathrm{~S}$ \\
\hline $6.61 \mathrm{M}$ \\
\hline $1.63 \mathrm{U}$ \\
\hline
\end{tabular}

\begin{tabular}{|r|l|l|l|}
\hline Ciphertext & $\begin{array}{l}\text { Dec. } \\
\text { Key } \\
(-)\end{array}$ & Plaintext & $\begin{array}{l}\text { Decrypted } \\
\text { message }\end{array}$ \\
\hline 4.75 & 1.7451 & $3.0049 \mathrm{~S}$ \\
\hline 3.21 & 1.5102 & $1.6998 \mathrm{U}$ \\
\hline 7.62 & 1.2509 & $6.3691 \mathrm{~N}$ \\
\hline 2.3 & 0.8775 & $1.4225 \mathrm{Y}$ \\
\hline 8.05 & 0.9078 & 7.1422 & $\mathrm{Space}$ \\
\hline 4.85 & 2.1305 & $2.7195 \mathrm{~A}$ \\
\hline 7.86 & 3.0497 & $4.8103 \mathrm{~L}$ \\
\hline 9.39 & 3.2823 & $6.1077 \mathrm{~B}$ \\
\hline 3.01 & 0.2861 & $2.7239 \mathrm{~A}$ \\
\hline 6.61 & 0.2320 & $6.378 \mathrm{~N}$ \\
\hline 1.63 & 0.1991 & $1.4309 \mathrm{Y}$ \\
\hline
\end{tabular}
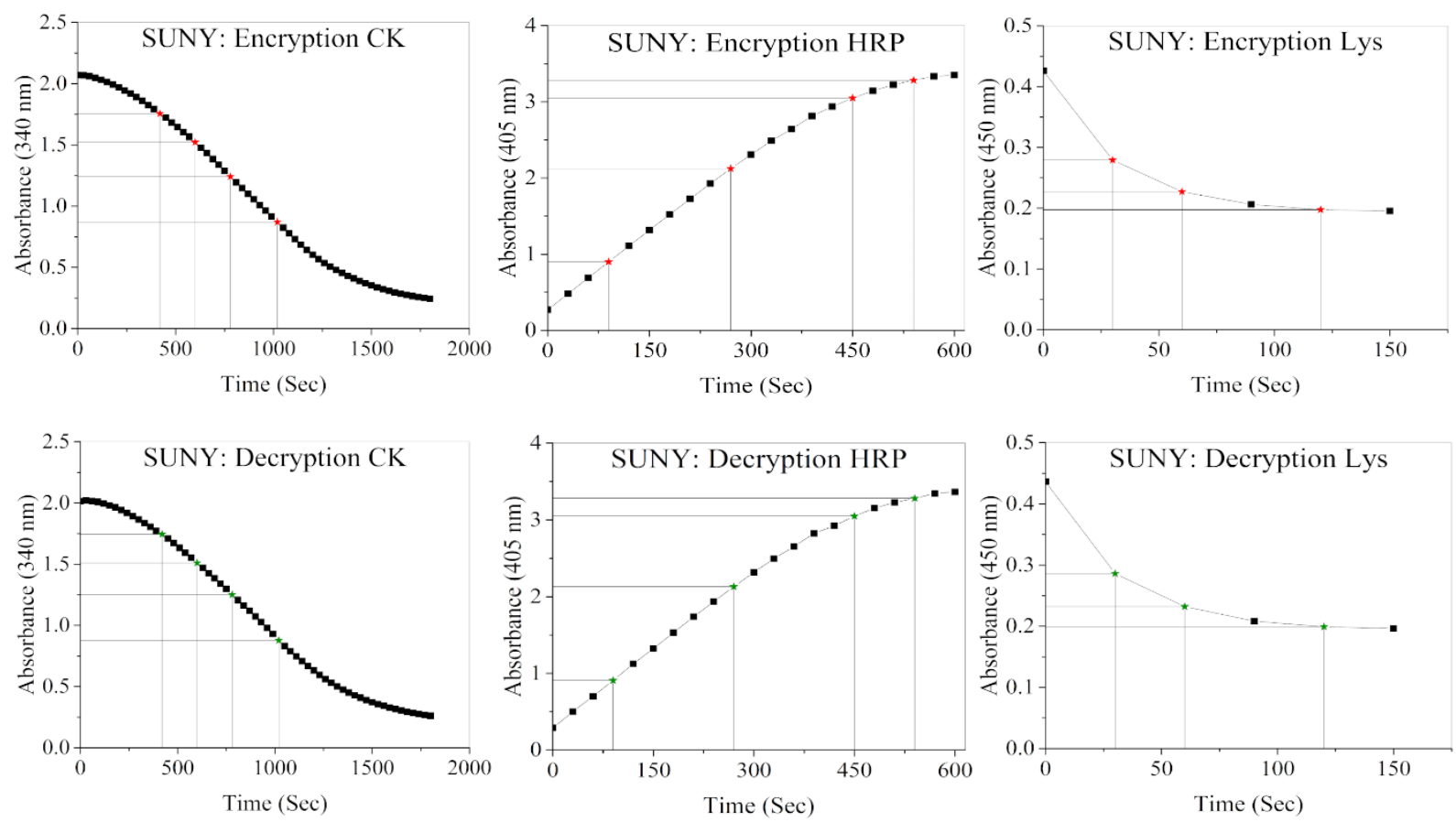


\section{Extra Message 3: SYNTHETIC BIOLOGY}

HRP, ALP, Lys

HRP:10 mU, $1 \mathrm{mM}$ substrates, Citrate Buffer $\mathrm{pH} 5.54,405 \mathrm{~nm}$, point/15 sec

ALP: $1 \mathrm{U}, 2 \mathrm{mM}$ pNPP, Carbonate buffer 6.44, 405nm, point/30 sec

Lys: $600 \mathrm{U}, 0.15 \mathrm{mg} / \mathrm{mL}$ cells, Citrate Buffer $\mathrm{pH} 5.54$, cut for plateau, $450 \mathrm{~nm}$, point $/ 15 \mathrm{sec}$

All: $30{ }^{\circ} \mathrm{C}, 5 \mathrm{~min}$

\begin{tabular}{|l|l|l|l|l|l|}
\hline Letter & $\begin{array}{l}\text { Numerical } \\
\text { (Table 1A) }\end{array}$ & $\begin{array}{l}\text { Time point } \\
\text { (Sec) }\end{array}$ & $\begin{array}{l}\text { Enc. } \\
\text { Key } \\
(+)\end{array}$ & Ciphertext (raw) & $\begin{array}{l}\text { Sent } \\
\text { ciphertext }\end{array}$ \\
\hline S & 2.99 & HRP 45 & 0.9437 & 3.9337 & 3.93 \\
\hline Y & 1.43 & ALP & 1.1882 & 2.6182 & 2.62 \\
\hline N & 6.38 & Lys 0 & 0.4184 & 6.7984 & 6.80 \\
\hline T & 1.17 & HRP 120 & 2.1110 & 3.281 & 3.28 \\
\hline H & 4.03 & ALP & 1.5850 & 5.6150 & 5.62 \\
\hline E & 0.65 & Lys 15 & 0.2982 & 0.9482 & 0.95 \\
\hline T & 1.17 & HRP 165 & 2.7028 & 3.8728 & 3.87 \\
\hline I & 1.95 & ALP & 2.0258 & 3.9758 & 3.98 \\
\hline C & 5.59 & Lys 30 & 0.2404 & 5.8304 & 5.83 \\
\hline Space & 7.15 & ALP & 2.9752 & 10.1252 & 10.13 \\
\hline B & 6.11 & HRP 195 & 2.9962 & 9.1062 & 9.11 \\
\hline I & 1.95 & Lys 45 & 0.2115 & 2.1615 & 2.16 \\
\hline O & 2.21 & ALP & 3.1590 & 5.3690 & 5.37 \\
\hline L & 4.81 & HRP 240 & 3.2835 & 8.0935 & 8.09 \\
\hline O & 2.21 & Lys 60 & 0.1961 & 2.4061 & 2.41 \\
\hline G & 3.77 & ALP & 3.2790 & 7.0490 & 7.05 \\
\hline Y & 1.43 & HRP 270 & 3.3461 & 4.7761 & 4.78 \\
\hline & & & & & \\
\hline
\end{tabular}

\begin{tabular}{|l|l|}
\hline Ciphertext & $\begin{array}{l}\text { Direct } \\
\text { translation }\end{array}$ \\
\hline 3.93 & $\mathrm{H}$ \\
\hline 2.62 & $\mathrm{~A}$ \\
\hline 6.80 & $\cdot$ \\
\hline 3.28 & $\mathrm{D}$ \\
\hline 5.62 & $\mathrm{C}$ \\
\hline 0.95 & $\mathrm{R}$ \\
\hline 3.87 & $\mathrm{G}$ \\
\hline 3.98 & $\mathrm{H}$ \\
\hline 5.83 & $\mathrm{~V}$ \\
\hline 10.13 & 0 (too high) \\
\hline 9.11 & 8 \\
\hline 2.16 & $\mathrm{O}$ \\
\hline 5.37 & $\mathrm{X}$ \\
\hline 8.09 & 4 \\
\hline 2.41 & $\mathrm{P}$ \\
\hline 7.05 & Space \\
\hline 4.78 & $\mathrm{~L}$ \\
\hline & \\
\hline
\end{tabular}

\begin{tabular}{|l|l|l|l|}
\hline Ciphertext & $\begin{array}{l}\text { Dec. } \\
\text { Key } \\
(-)\end{array}$ & Plaintext & $\begin{array}{l}\text { Decrypted } \\
\text { message }\end{array}$ \\
\hline 3.93 & 0.9368 & 2.9932 & S \\
\hline 2.62 & 1.2115 & 1.4085 & Y \\
\hline 6.80 & 0.4134 & 6.3866 & N \\
\hline 3.28 & 2.1327 & 1.1473 & T \\
\hline 5.62 & 1.6245 & 3.9955 & H \\
\hline 0.95 & 0.2931 & 0.6569 & E \\
\hline 3.87 & 2.7088 & 1.1612 & T \\
\hline 3.98 & 2.0300 & 1.9500 & I \\
\hline 5.83 & 0.2373 & 5.5927 & C \\
\hline 10.13 & 2.9769 & 7.1531 & Space \\
\hline 9.11 & 2.9987 & 6.1113 & B \\
\hline 2.16 & 0.2105 & 1.9495 & I \\
\hline 5.37 & 3.1570 & 2.2130 & O \\
\hline 8.09 & 3.2760 & 4.8140 & L \\
\hline 2.41 & 0.1964 & 2.2136 & O \\
\hline 7.05 & 3.2807 & 3.7693 & G \\
\hline 4.78 & 3.3461 & 1.4339 & Y \\
\hline & & & \\
\hline
\end{tabular}
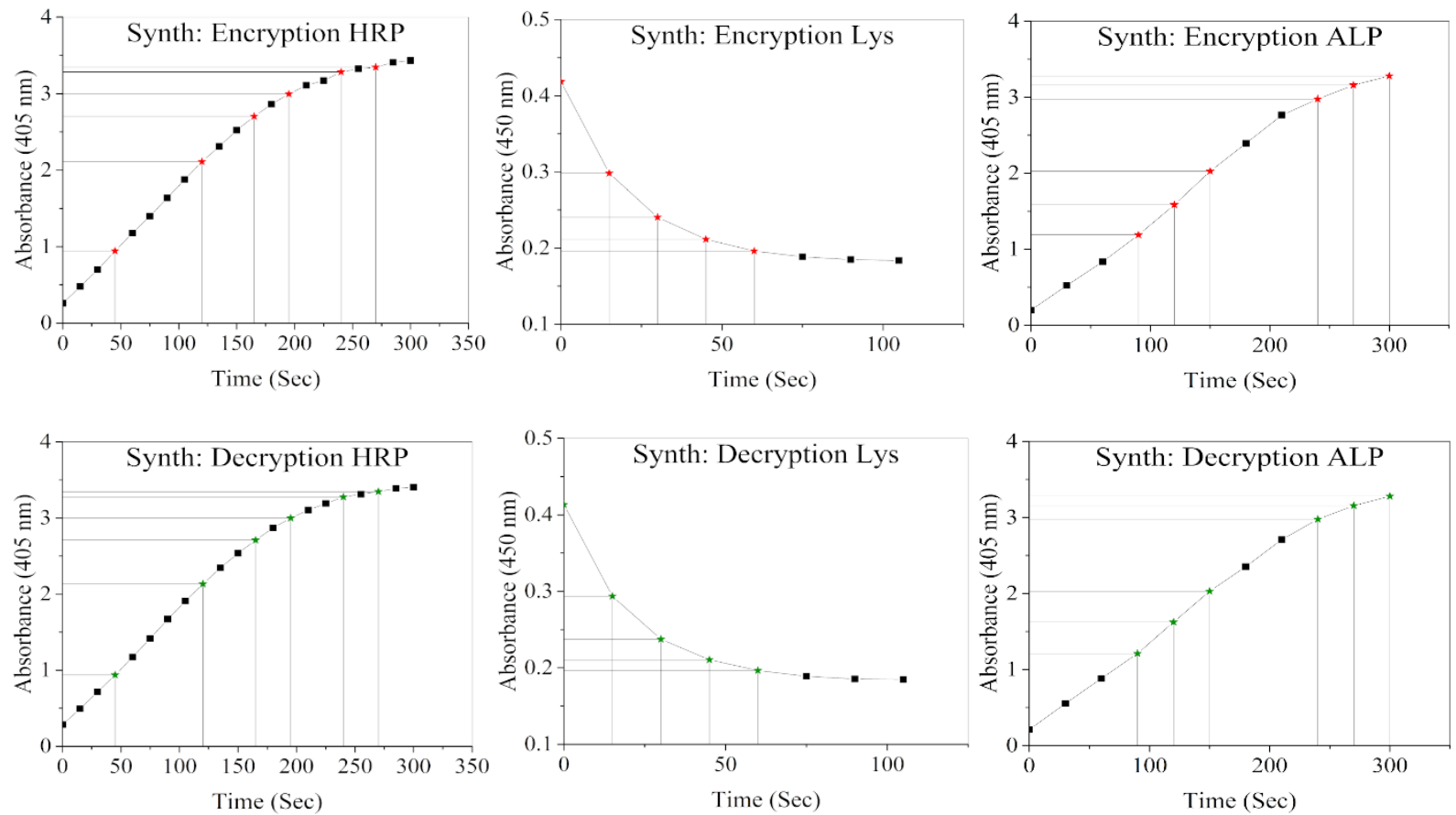